\title{
Japanese gastric cancer treatment guidelines 2010 (ver. 3)
}

\author{
Japanese Gastric Cancer Association
}

Published online: 14 May 2011

(c) The International Gastric Cancer Association and The Japanese Gastric Cancer Association 2011

The description of tumor status (T/N/M and stage) in this guideline is based on the 3rd English edition of the Japanese Classification of Gastric Carcinoma [1] which is identical to that in the 7th edition of the International Union Against Cancer (UICC)/TNM.

\section{Treatments}

1.1 Algorithm of standard treatments

to be recommended in clinical practice

The algorithm is shown on the following page.

\subsection{Investigational treatments}

The following treatments show promise but are as yet to be established as standard. They should be prospectively evaluated in appropriate clinical research settings. Patient consent for investigational treatments should be sought and the rationale behind them given (Refer to the Sect. 6 "Commentary on investigational treatments" for details). The following constitute investigational treatments:

- Endoscopic submucosal dissection under expanded criteria

The online version of the prefatory article referred to in this article can be found under doi:10.1007/s10120-011-0040-6.

English edition editors: Takeshi Sano $(\bowtie)$, Yasuhiro Kodera. e-mail: takeshi.sano@jfcr.or.jp

Japanese Gastric Cancer Association ( $\square$ )

Association Office, First Department of Surgery,

Kyoto Prefectural University of Medicine, Kawaramachi,

Kamigyo-ku, Kyoto 602-0841, Japan

e-mail: jgca@koto.kpu-m.ac.jp
- Laparoscopic gastrectomy

- Local tumor resection

- Neoadjuvant chemotherapy

- Adjuvant chemotherapy using agents other than S-1

- Neoadjuvant chemoradiotherapy

- Adjuvant chemoradiotherapy

- Debulking surgery.

\section{Surgery}

2.1 Types and definitions of gastric surgery

\subsubsection{Curative surgery}

2.1.1.1 Standard gastrectomy Standard gastrectomy is the principal surgical procedure performed with curative intent. It involves resection of at least two-thirds of the stomach with a D2 lymph node dissection.

2.1.1.2 Non-standard gastrectomy In non-standard gastrectomy, the extent of gastric resection and/or lymphadenectomy is altered according to the tumor characteristics.

2.1.1.2.1 Modified surgery The extent of gastric resection and/or lymphadenectomy is reduced compared to standard surgery.

2.1.1.2.2 Extended surgery (1) Gastrectomy with combined resection of adjacent involved organs. (2) Gastrectomy with extended lymphadenectomy exceeding D2.

\subsubsection{Non-curative surgery}

2.1.2.1 Palliative surgery Urgent presentations with symptoms of bleeding or obstruction may develop in patients with advanced gastric cancer with unresectable 


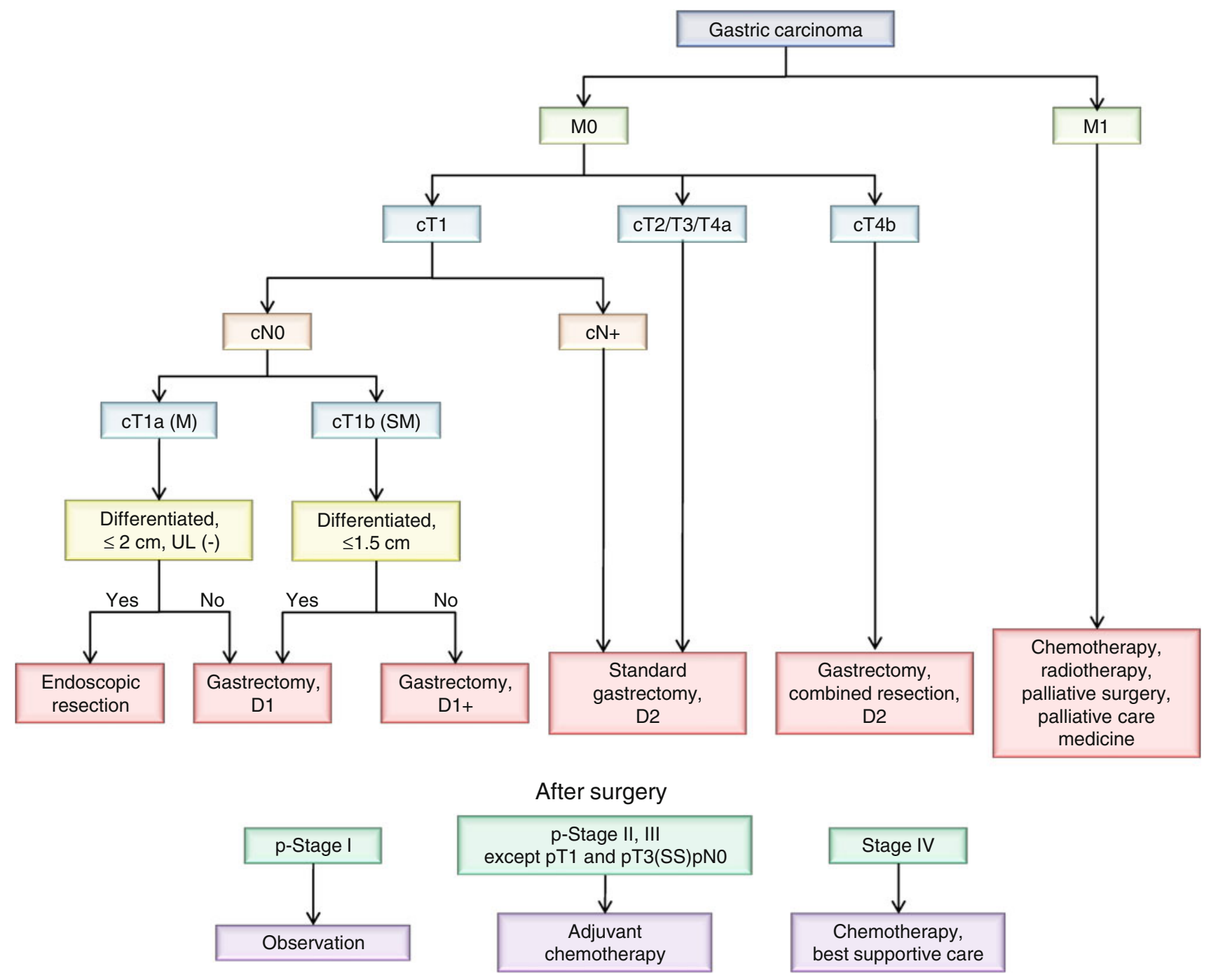

metastases. Palliative surgery to relieve symptoms is recommended as an option for stage IV gastric cancer, provided that the patient is fit. Palliative gastrectomy or gastrojejunostomy is selected depending on the resectability of the primary tumor and/or surgical risks. Stomachpartitioning gastrojejunostomy has been reported to result in superior function compared to simple gastrojejunostomy [2].

2.1.2.2 Reduction surgery The role of gastrectomy is unclear in patients with advanced gastric cancer with unresectable metastatic disease in the absence of urgent symptoms such as bleeding or obstruction. Reduction surgery aims to prolong survival or to delay the onset of symptoms by reducing tumor volume. To date there is no evidence demonstrating the benefit of reduction surgery for gastric cancer and it should only be considered in an investigational setting. A randomized controlled trial to explore this issue is underway as an international cooperative trial (REGATTA, JCOG0705/KGCA01) [3].
2.2 Extent of gastric resection

\subsubsection{Gastric resections}

Gastric resections for gastric cancer are listed below in the order of the stomach volume to be resected.

- Total gastrectomy

- Distal gastrectomy

- Pylorus-preserving gastrectomy (PPG)

- Proximal gastrectomy

- Segmental gastrectomy

- Local resection

- Non-resectional surgery (bypass surgery, gastrostomy, jejunostomy).

\subsubsection{Determination of gastric resection}

2.2.2.1 Resection margin A sufficient resection margin should be ensured when determining the resection line in 
gastrectomy with curative intent. A proximal margin of at least $3 \mathrm{~cm}$ is recommended for $\mathrm{T} 2$ or deeper tumors with an expansive growth pattern (Types 1 and 2 ) and $5 \mathrm{~cm}$ is recommended for those with infiltrative growth pattern (Types 3 and 4). When these rules cannot be observed, it is advisable to examine the proximal resection margin by frozen section. For tumors invading the esophagus, a $5-\mathrm{cm}$ margin is not necessarily required, but frozen section examination of the resection line is desirable to ensure an $\mathrm{R} 0$ resection.

For T1 tumors, a gross resection margin of $2 \mathrm{~cm}$ should be obtained. When the tumor border is unclear, preoperative endoscopic marking, by clips, of the tumor border based on biopsy results will be helpful for decision-making regarding the resection line.

2.2.2.2 Selection of gastrectomy The standard surgical procedure for clinically node-positive $(\mathrm{cN}+)$ or $\mathrm{T} 2-\mathrm{T} 4 \mathrm{a}$ tumors is either total or distal gastrectomy. Distal gastrectomy is selected when a satisfactory proximal resection margin (see above) can be obtained. Pancreatic invasion by tumor requiring pancreaticosplenectomy necessitates total gastrectomy regardless of the tumor location. Total gastrectomy with splenectomy should be considered for tumors that are located along the greater curvature and harbor metastasis to no. $4 \mathrm{sb}$ lymph nodes, even if the primary tumor could be removed by distal gastrectomy. For adenocarcinoma located on the proximal side of the esophagogastric junction, esophagectomy and proximal gastrectomy with gastric tube reconstruction should be considered, similarly to surgery for esophageal cancer.

For $\mathrm{cT} 1 \mathrm{cN} 0$ tumors, gastric resection can be modified as follows according to tumor location.

- Pylorus-preserving gastrectomy (PPG) for tumors in the middle portion of the stomach with the distal tumor border at least $4 \mathrm{~cm}$ proximal to the pylorus.

- Proximal gastrectomy for proximal tumors where more than half of the distal stomach can be preserved.

Segmental gastrectomy and local resection are still regarded as investigational treatments.

\subsection{Lymph node dissection}

\subsubsection{Extent of lymph node dissection}

The extent of systematic lymphadenectomy is defined as follows according to the type of gastrectomy indicated. When the lymphadenectomy performed does not comply with the D level criteria (either when lymph nodes outside the requirement for the $\mathrm{D}$ criteria are resected or when nodal dissection is insufficient to fulfill the criteria), the lymph node station that has been dissected or omitted should be specified, as in the following examples: D1 (+No. 8a), D2 (-No. 10). When reporting the data to construct a formal database, only the $\mathrm{D}$ level that has been completely resected should be provided.

\subsubsection{Total gastrectomy}

D0: Lymphadenectomy less than D1

D1: $\quad$ Nos. $1-7$

D1+: $\quad$ D1 + Nos. 8a, 9, 11p

D2: $\quad$ D1 + Nos. 8a, 9, 10, 11p, 11d, 12a.

For tumors invading the esophagus, D1+ includes No. $110^{1}$, D2 includes Nos. 19, 20, 110, and 111.

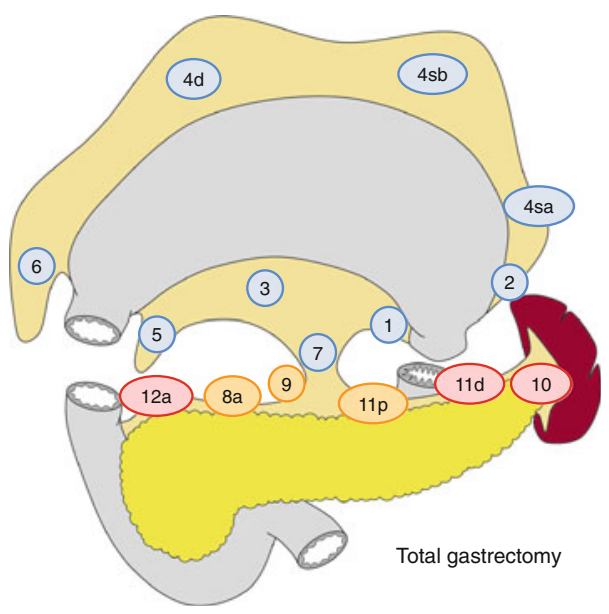

\subsubsection{Distal gastrectomy}

D0: Lymphadenectomy less than D1

D1: $\quad$ Nos. $1,3,4 \mathrm{sb}, 4 \mathrm{~d}, 5,6,7$

D1+: $\quad$ D1 + Nos. 8a, 9

D2: $\quad$ D1 + Nos. 8a, 9, 11p, 12a.

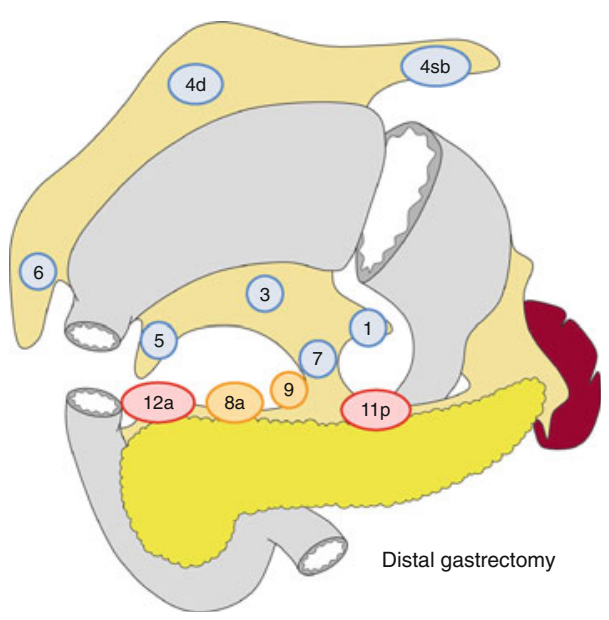

${ }^{1}$ No. 110 lymph nodes (lower thoracic para-esophageal nodes) in gastric cancer invading the esophagus are those attached to the lower part of the esophagus that is removed to obtain a sufficient resection margin. 


\subsubsection{Pylorus-preserving gastrectomy}

D0: Lymphadenectomy less than D1

D1: $\quad$ Nos. 1, 3, 4sb, 4d, 6, 7

D1+: $\quad$ 1 + Nos. 8a, 9 .

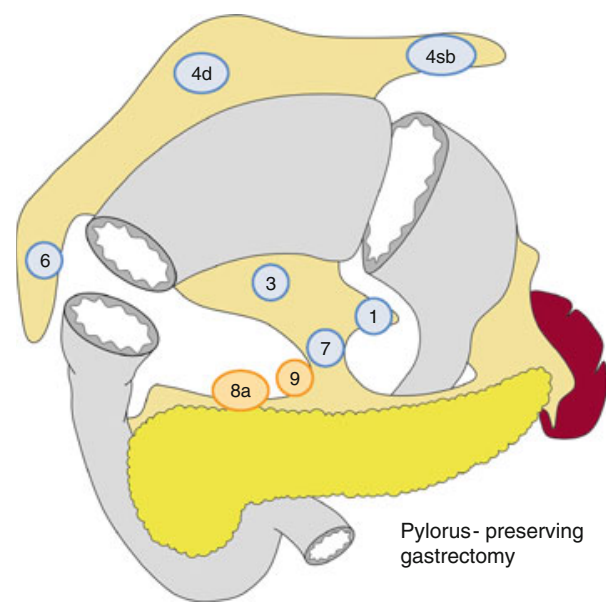

\subsubsection{Proximal gastrectomy}

D0: Lymphadenectomy less than D1

D1: $\quad$ Nos. 1, 2, 3a, 4sa, 4sb, 7

D1+: $\quad$ D1 + Nos. 8a, 9, 11p.

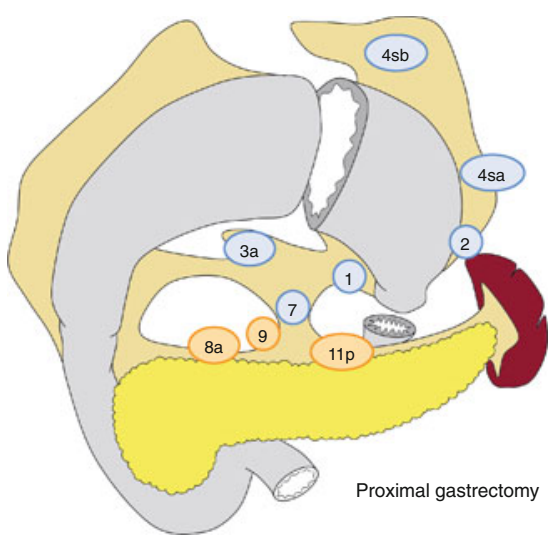

For tumors invading the esophagus, D1+ includes node No. 110 (see footnote 1 on the preceding page).

\subsubsection{Indications for lymph node dissection}

In principle, a D1 or a D1+ lymphadenectomy is indicated for cT1N0 tumors, and D2 is indicated for $\mathrm{cN}+$ or cT2-T4 tumors. Because the pre- and intraoperative diagnoses of lymph node metastases remain unreliable, a D2 lymphadenectomy should be performed whenever nodal involvement is suspected.

2.3.2.1 D1 lymphadenectomy A D1 lymphadenectomy is indicated for $\mathrm{T} 1 \mathrm{a}$ tumors that do not meet the criteria for endoscopic mucosal resection (EMR)/ endoscopic submucosal resection (ESD), and for cT1bN0 tumors that are histologically of differentiated type and $1.5 \mathrm{~cm}$ or smaller in diameter.

2.3.2.2 D1+ lymphadenectomy A D1+ lymphadenectomy is indicated for cT1N0 tumors other than the above.

2.3.2.3 D2 lymphadenectomy A D2 lymphadenectomy is indicated for potentially curable T2-T4 tumors, as well as cT1N+ tumors. The role of splenectomy for complete resection of No. 10 and No. 11 nodes has long been controversial and the final results of randomized trial JCOG 0110 are awaited [4]. In the meantime, complete clearance of No. 10 nodes by splenectomy should be considered for potentially curable T2-T4 tumors invading the greater curvature of the upper stomach.

2.3.2.4 D2 + lymphadenectomy Gastrectomy with extended lymphadenectomy beyond D2 is classified as a nonstandard gastrectomy. Its role has been discussed as follows:

- The benefit of prophylactic para-aortic lymphadenectomy was denied by the Japanese randomized controlled trial (RCT) JCOG 9501 [5].

- Although an R0 resection may be possible for tumors with para-aortic nodal involvement without other noncurative factors, the prognosis of this population is poor.

- The role of No. 14v lymphadenectomy in distal gastric cancer is controversial. Dissection of node No. 14v had been a part of D2 gastrectomy defined by the previous edition of the Japanese classification [6], but it has been excluded from the current edition. However, D2 $(+$ No. 14v) may be beneficial in tumors with apparent metastasis to the No. 6 nodes.

- Involvement of No. 13 nodes is defined as M1 in the current version of the Japanese classification. However, D2 (+ No. 13) lymphadenectomy may be an option in a potentially curative gastrectomy for tumors invading the duodenum [7].

\subsection{Miscellaneous}

\subsubsection{Vagal nerve preservation}

It is reported that preservation of the hepatic branch of the anterior vagus and/or the celiac branch of the posterior 
vagus contributes to improving the postoperative quality of life through reducing post-gastrectomy gallstone formation, diarrhea, and/or weight loss. In PPG, the hepatic branch should be preserved to maintain pyloric function.

\subsubsection{Omentectomy}

Removal of the greater omentum is usually integrated in the standard gastrectomy for T3 (SS) or deeper tumors. For T1/T2 tumors, the omentum more than $3 \mathrm{~cm}$ away from the gastroepiploic arcade may be preserved.

\subsubsection{Bursectomy}

For tumors penetrating the serosa of the posterior gastric wall, bursectomy (removal of the inner peritoneal surface of the bursa omentalis) may be performed with the aim of removing microscopic tumor deposits in the lesser sac. There is no evidence that bursectomy reduces peritoneal or local recurrence, and it should be avoided in T1/T2 tumors to prevent injury to the pancreas and/or adjacent blood vessels.

A small-scale RCT recently suggested a survival benefit for bursectomy in T3/T4a tumors. A large-scale multiinstitutional RCT has been commenced to address this issue (JCOG 1001).

\subsubsection{Combined resection of adjacent organ $(s)$}

For tumors in which the primary or metastatic lesion directly invades adjacent organs, combined resection of the involved organ may be performed in order to obtain an R0 resection.

\subsubsection{Approaches to the lower esophagus}

For gastric cancers invading less than $3 \mathrm{~cm}$ of the distal esophagus, a transhiatal abdominal approach is recommended [8]. Where a greater length of esophagus is involved a transthoracic approach should be considered if the surgery is potentially curative.

\subsubsection{Laparoscopic surgery}

Laparoscopic surgery has been increasingly employed, largely for T1 tumors, as it has some advantages over open surgery in terms of minimal invasiveness. However, it is technically demanding and solid evidence regarding safety and long-term outcome remains lacking. It should thus be considered as an investigational treatment and should be evaluated further in clinical research settings (Refer to the Sect. 6.2).

\subsection{Reconstruction after gastrectomy}

The following reconstruction methods are usually employed. Each has advantages and disadvantages. The functional benefits of pouch reconstruction are yet to be established.

\subsubsection{Total gastrectomy}

- Roux-en-Y esophagojejunostomy

- Jejunal interposition

- Double tract method

\subsubsection{Distal gastrectomy}

- Billroth I gastroduodenostomy

- Billroth II gastrojejunostomy

- Roux-en-Y gastrojejunostomy

- Jejunal interposition

\subsubsection{Pylorus-preserving gastrectomy}

- Gastro-gastrostomy

\subsubsection{Proximal gastrectomy}

- Esophagogastrostomy

- Jejunal interposition

- Double tract method.

\section{Endoscopic resection}

3.1 Methods of endoscopic resection

\subsubsection{Endoscopic mucosal resection (EMR)}

The lesion, together with the surrounding mucosa, is lifted by submucosal injection of saline (normo- or hypertonic) and removed using a high-frequency steel snare.

\subsubsection{Endoscopic submucosal dissection (ESD)}

The mucosa surrounding the lesion is circumferentially incised using a high-frequency electric knife (usually 
insulation-tipped) and the submucosal layer is dissected from the proper muscle layer.

\subsection{Handling of endoscopically resected specimens}

\subsubsection{Handling of resected specimens}

The resected specimens should be handled according to the rules described in the Japanese classification [1].

\subsubsection{Definitions of differentiated-type and undifferentiated-type carcinoma}

The tumor biopsy specimens and endoscopically resected tumors are histologically classified as either differentiated type or undifferentiated type. The former includes papillary adenocarcinoma (pap) and tubular adenocarcinoma (tub1, tub2), and the latter includes poorly differentiated adenocarcinoma (por1, por2), signet-ring cell carcinoma (sig), and mucinous adenocarcinoma (muc) (refer to the Japanese classification [1]).

\subsubsection{Histological predominance and intratumoral ulcerative findings $(U L)$}

A tumor consisting of components of both differentiatedand undifferentiated-type carcinoma is classified according to the quantitative predominance. Different histological types seen in a tumor are recorded according to the quantitative predominance, e.g., tub2 $>$ tub1. Diagnosis of $\mathrm{UL}(+)$ is principally based on the histological evidence of ulcerative findings. However, endoscopic and/or radiological evidence should also be taken into consideration when making a conclusive diagnosis.

\subsection{Indication for endoscopic resection}

\subsubsection{Principles of indication}

Endoscopic resection is considered for tumors which have a very low possibility of lymph node metastasis and are suitable for en-bloc resection.

Since the compilation of the first version of these Guidelines, two independent sets of indications have been provided for endoscopic resection; an absolute indication for standard EMR/ESD, and an expanded indication for ESD as an investigational treatment. Although the latter should appear in the Sect. 6 "Commentary on investigational treatments", it may be more apt to mention it here along with the absolute indication, firstly because expert endoscopists today almost routinely perform ESD under the expanded criteria outside the clinical trial setting, and secondly because the paramount importance of issues such as the assessment of curability through adequate evaluation of the resected specimen, and the follow-up strategy, is common to both the standard EMR/ESD and ESD under the expanded indication. Again, the users of these guidelines are reminded that the evidence regarding the curability of the latter technique (i.e., ESD under the expanded criteria) remains insufficient, and the procedure should be offered with caution.

\subsubsection{Tumors indicated for endoscopic resection as a standard treatment (absolute indication)}

EMR or ESD is indicated as a standard treatment for the following tumor.

- A differentiated-type adenocarcinoma without ulcerative findings (UL $(-)$ ), of which the depth of invasion is clinically diagnosed as T1a and the diameter is $\leq 2 \mathrm{~cm}$.

\subsubsection{Tumors indicated for endoscopic resection as an investigational treatment (expanded indication)}

Tumors of the following categories have a very low possibility of lymph node metastasis [9, 10]. Endoscopic resection for these tumors is regarded as an investigational treatment. Not EMR but ESD should be employed.

- Tumors clinically diagnosed as T1a and:

(a) of differentiated-type, UL(-), but $>2 \mathrm{~cm}$ in diameter

(b) of differentiated-type, $\mathrm{UL}(+)$, and $\leq 3 \mathrm{~cm}$ in diameter

(c) of undifferentiated-type, $\mathrm{UL}(-)$, and $\leq 2 \mathrm{~cm}$ in diameter.

\subsubsection{Local recurrence after EMR/ESD}

Local mucosal recurrence after EMR/ESD for tumors fulfilling the absolute indication could be treated by another ESD. However, given the paucity of evidence regarding the validity of repeat ESD, it should be performed as a part of investigational therapy.

\subsection{Curability of endoscopic resection}

Two factors should be considered for curability assessment: completeness of the primary tumor removal and nil possibility of lymph node metastasis.

\subsubsection{Curative resection}

The resection is judged as curative when all of the following conditions are fulfilled: en-bloc resection, tumor 
size $\leq 2 \mathrm{~cm}$, histologically of differentiated-type, pT1a, negative horizontal margin (HM0), negative vertical margin (VM0), and no lymphovascular infiltration $(\mathrm{ly}(-), \mathrm{v}(-))$.

\subsubsection{Curative resection for tumors of expanded indications}

The resection is considered as curative when all of the following conditions are fulfilled:

- En-bloc resection, HM0, VM0, ly(-), v(-), and:

(a) tumor size $>2 \mathrm{~cm}$, histologically of differentiatedtype, pT1a, UL(-), or

(b) tumor size $\leq 3 \mathrm{~cm}$, histologically of differentiatedtype, pT1a, UL $(+)$, or

(c) tumor size $\leq 2 \mathrm{~cm}$, histologically of undifferentiatedtype, pT1a, UL(-), or

(d) tumor size $\leq 3 \mathrm{~cm}$, histologically of differentiated-type, pT1b (SM1, $<500$ micron from the muscularis mucosae).

As the evidence is still insufficient for differentiatedtype tumors associated with some areas of undifferentiatedtype histology, the following resections are regarded as non-curative for the time being, and the addition of surgical treatments should be recommended.

- Areas of undifferentiated-type carcinoma that exceed $2 \mathrm{~cm}$ in (a) above.

- Any component of undifferentiated-type carcinoma in (b) above.

- Undifferentiated-type component in the submucosal invasion in (d) above.

\subsubsection{Non-curative resection}

Resection that does not satisfy any of the above criteria is considered non-curative.

\subsection{Treatments after endoscopic resection}

\subsubsection{Treatments after curative resection}

Helicobacter pylori should be tested for, and if positive, should be eradicated [11]. Follow-up with annual or biannual endoscopy is recommended.

\subsubsection{Treatments after curative resection for tumors of expanded indications}

Helicobacter pylori should be examined, and if positive, should be eradicated. Follow-up with abdominal ultrasonography or computed tomography (CT) scan as well as annual or biannual endoscopy is recommended.

\subsubsection{Treatment after non-curative resection}

Surgical treatment should be performed after non-curative resection. However, as the following cases actually carry a very low risk of harboring lymph node metastasis, nonsurgical treatments such as repeated ESD, endoscopic coagulation using LASER or argon-plasma coagulator, or close observation expecting a burn effect of the initial ESD could be proposed as alternatives and delivered upon the patient's informed consent.

- En-bloc resection of a differentiated-type carcinoma with positive horizontal margin (HM1) as the only noncurative factor.

- Piecemeal resection of a differentiated-type carcinoma satisfying all other criteria.

When these cases come from the category (b) or (d) in the Sect. 3.4.2, the size of the residual mucosal lesion should be assessed. If the sum of the length of the resected and residual lesions exceeds $3 \mathrm{~cm}$, surgery is indicated. When the positive horizontal margin or the piecemeal resection margin involves part of the submucosal invasion in category (d), surgery is indicated.

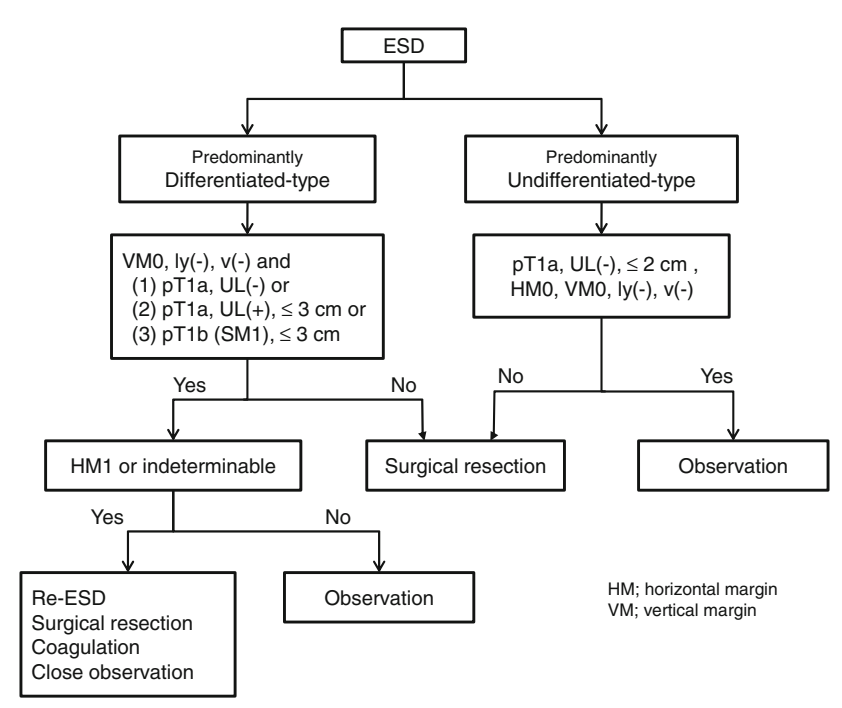

\section{Chemotherapy}

Although recent advances in chemotherapy have achieved considerable tumor regression in many cases of unresectable/recurrent gastric cancer, these responses have not ultimately led to complete cure. The median survival time achieved in clinical trials for the disease at this stage remains to be 6-13 months. The current goal of chemotherapy therefore is to delay the appearance of 
disease-related symptoms and/or to prolong survival. The survival benefit of chemotherapy has been proven in RCTs comparing chemotherapy with best supportive care in patients with unresectable gastric cancer [12-14]. Although very rare, some patients with advanced disease even survive for more than 5 years after chemotherapy alone. Thus, chemotherapy is the treatment to be primarily considered for unresectable/recurrent gastric cancer.

\subsection{Principles of indication}

Chemotherapy is indicated for patients with unresectable or recurrent disease, or those after non-curative R2 resection, whose general condition and major organ functions are preserved: to be specific, patients of performance status 0-2, with unresectable T4b disease, extensive nodal disease, hepatic metastases, peritoneal dissemination, or other M1 disease.

\subsection{Recommended regimens for Japanese patients}

Based on the evidence obtained by phase 3 trials, the following regimens are recommended for clinical practice in Japan. All other regimens should be considered as investigational at present. Establishment of new solid evidence will be announced on the website of the Japanese Gastric Cancer Association.

\subsubsection{First-line regimen}

- S-1 + cisplatin

The recommendation is based on the results of the SPIRITS trial [15] and the JCOG 9912 trial [16]. Irinotecan + cisplatin and S-1 + irinotecan combinations are not recommended as the first-line regimen because they did not show significant superiority over 5-fluorouracil (FU) alone and S-1 alone, respectively, in the randomized trials [17].

Use of S-1 + cisplatin should be carefully decided in patients with limited oral intake, moderate volume of ascites, intestinal stenosis/obstruction, and/or in the elderly. When S-1 + cisplatin is considered as inappropriate, either S-1 or 5-FU should be delivered as a single agent depending on the condition of the patient.

\subsubsection{Second-line regimen}

- No single recommended regimen

To date, there is no evidence of survival benefit with second-line chemotherapy for gastric cancer. In patients with good performance status, second-line chemotherapy may serve to control the cancer-related symptoms. Usually agents that were not used in the previous chemotherapy are selected. Taxane- or irinotecan-based regimens are being evaluated in randomized trials.

\subsubsection{Chemotherapy for peritoneal disease}

- No regimen specific to this condition

Several reports showed the efficacy of methotrexate + 5-FU or taxanes on this disease status that is peculiar to gastric cancer. However, methotrexate +5 -FU did not show significant superiority over 5-FU alone in an RCT [18] (JCOG 0106) for peritoneal disease, and therefore is not recommended.

\section{Adjuvant chemotherapy}

Adjuvant chemotherapy is delivered with the intention to reduce recurrence by controlling residual tumor cells following curative resection. Various regimens had been tested in numerous clinical trials in Japan without producing solid evidence in support of adjuvant chemotherapy until the ACTS-GC trial [19] showed the efficacy of S-1 in 2006.

\subsection{Indications}

The patients tested in the ACTS-GC trial were those with a tumor of pathological stage II, IIIA, or IIIB excluding T1, defined in the previous 13th edition of the Japanese classification, who had undergone R0 gastrectomy with D2 or greater lymphadenectomy. Accordingly, this Guideline recommends S-1 adjuvant chemotherapy for the same population.

Simulation has revealed that "patients of stage II, IIIA or IIIB, except for T1" defined in the 13th edition of the Japanese classification correspond to "patients of stage IIA, IIB, IIIA, IIIB, or IIIC, except for T1 and T3(SS)/N0" defined in the 14th edition, which is identical to the 7th edition of the International Union Against Cancer (UICC)/ TNM classification.

\subsection{Administration schedule}

$\mathrm{S}-1$ is to be started within 6 weeks from surgery, after sufficient recovery from the intervention. A 6-week cycle consisting of 4 weeks of daily oral administration of S-1 at a dose of $80 \mathrm{mg} / \mathrm{m}^{2}$ followed by 2 weeks of rest is repeated during the first year after surgery. 


\section{Commentary on investigational treatments}

The following treatments show promise but are yet to be established as standard treatment. They should be prospectively evaluated in appropriate clinical research settings. It is advised that informed consent is obtained from patients, ensuring that they understand the rationale for investigational treatments.

\subsection{Endoscopic submucosal dissection by expanded criteria}

Refer to the Sect. 3.3.

\subsection{Laparoscopic gastrectomy}

The number of laparoscopic gastrectomies is increasing in Japan and, according to the survey carried out by the Japan Society for Endoscopic Surgery and the statistical database of the Ministry of Health, Labor and Welfare of Japan, it is estimated that a laparoscopic approach is employed in about $20 \%$ of gastric cancer surgeries. However, the benefit of this potentially minimally invasive procedure has only been shown by small comparative studies $[20,21]$ and the evidence is still weak for the approach to be considered as a standard procedure in daily practice. Two RCTs to compare long-term survival after open and laparoscopic gastrectomy for early-stage cancer are currently ongoing in Japan and Korea (JCOG 0912 and KLASS trials) and the results are awaited.

\subsection{Local tumor resection}

Local tumor resection, usually wedge resection, was developed as a minimally invasive method to be placed intermediately between gastrectomy and endoscopic resection. However, since the expanded indications for endoscopic resection have been introduced, its indication has become limited to poor-risk patients. Local tumor resection will be reevaluated when the diagnostic value of sentinel node navigation is established.

\subsection{Neoadjuvant chemotherapy}

Neoadjuvant chemotherapy is delivered with the aim of controlling microscopic metastasis and/or downstaging/ downsizing the tumor and thereby enhancing the surgical curability. Although an improvement in the survival of chemotherapy-responders has been reported, the benefit for all patients on an intention-to-treat basis has not yet been established in Japan. A randomized trial is currently ongoing for large type-3 and type-4 tumors (JCOG 0501).
The following cases could be the subject of prospective studies.

- R0 resection is possible but the recurrence risk is relatively high: cStage IIIA-IIIC (cT4, cN+, no peritoneal/hepatic metastasis).

- R0/R1 resection is deemed possible but the prognosis is poor: extensive nodal disease; large type- 3 or type- 4 tumors.

\subsection{Adjuvant chemotherapy using agents other than S-1}

In the West, both postoperative adjuvant chemoradiation [22] and perioperative chemotherapy [23] were shown to be superior to treatment with surgery alone in large-scale randomized trials. However, evidence obtained in these trials cannot be applied to Japanese patients, because the standards of surgery performed and actual outcomes of the study populations in these trials were vastly different from those in a similar population in Japan. Apart from the ACTS-GC trial of S-1, the only randomized trial that has shown a survival benefit of adjuvant chemotherapy over surgery in Japan is the NSAS-GC, in which high-dose uracil-tegafur (UFT) was administered for 16 months [24]. Because the subjects in this trial were limited to those with T2/N1-2 disease and the number of cases was small $(n=190)$ due to slow accrual, the treatment could not be considered as a standard to be widely applied for gastric cancer patients. UFT may be an alternative for the T2/N1-2 population when the patient cannot tolerate $\mathrm{S}-1$.

The ACTS-GC trial revealed that the outcome of Stage III patients remained unsatisfactory even when adjuvant chemotherapy with S-1 was added to adequate surgery. Multimodal strategy with greater efficacy will be the subject of future randomized trials for this population.

\subsection{Neoadjuvant chemoradiotherapy}

Neoadjuvant chemoradiotherapy is being evaluated in Western clinical trials: for cardiac and lower esophageal carcinomas in Europe, and for gastric carcinomas in the United States. High rates of histological complete response (20-30\%) have been reported in the American phase II trials [25], but no randomized study has been conducted to date to address the survival benefit of this modality.

Patients with gastric cancer of cStage II-III can be the subject of clinical studies. Absence of peritoneal disease should be confirmed by staging laparoscopy.

\subsection{Adjuvant chemoradiation therapy}

Adjuvant chemoradiation therapy has become the standard in the United States since the Intergroup Study 0116 
showed the survival benefit of this therapy when compared with surgery alone. However, the evidence was obtained in circumstances where lymphadenectomy was limited (D0/ D1) and radiation therapy was widely available. In Japan, where standardized D2 lymphadenectomy provides good local tumor control, there is no evidence that chemoradiation therapy improves survival. A randomized controlled trial is ongoing in Korea (ARTIST trial) to evaluate the role of adjuvant chemoradiation therapy following D2 lymphadenectomy, and the results are awaited.

\subsection{Reduction surgery}

Reduction surgery is an attempt to prolong survival by reducing the tumor volume in patients with non-resectable M1 disease. Some retrospective studies showed favorable results from such attempts in gastric cancer, but these studies suffered from considerable selection biases and cannot be regarded as solid evidence in support of this strategy, given the efficacy of today's chemotherapy. As previous reports were consistent in that reduction surgery had no survival benefit in patients with multiple non-curable factors [26], only patients with a single non-curable factor should be eligible for clinical trials exploring this issue. A Japan/Korea cooperative RCT is currently ongoing to compare reduction surgery followed by chemotherapy with primary chemotherapy alone in patients with either of the following factors:unresectable hepatic metastases, peritoneal metastases, or para-aortic nodal metastases (REGATTA trial).

Acknowledgments We thank Dr. Hisashi Shinohara for his illustrations and Dr. Rachel Melhado for her English-language advice.

\section{References}

1. Japanese Gastric Cancer Association. Japanese classification of gastric carcinoma: 3rd English edition. Gastric Cancer 2011. doi: 10.1007s/10120-011-0041-5

2. Kaminishi M, Yamaguchi H, Shimizu N, et al. Stomach-partitioning gastrojejunostomy for unresectable gastric carcinoma. Arch Surg. 1997;132:184-7.

3. Fujitani K, Yang HK, Kurokawa Y, et al. Randomized controlled trial comparing gastrectomy plus chemotherapy with chemotherapy alone in advanced gastric cancer with a single non-curable factor: Japan Clinical Oncology Group study JCOG 0705 and Korea Gastric Cancer Association study KGCA01. Jpn J Clin Oncol. 2008;38:504-6.

4. Sano T, Yamamoto S, Sasako M. Randomized controlled trial to evaluate splenectomy in total gastrectomy for proximal gastric carcinoma: Japan Clinical Oncology Group study JCOG 0110-MF. Jpn J Clin Oncol. 2002;32:363-4.

5. Sasako M, Sano T, Yamamoto S, et al. D2 lymphadenectomy alone or with para-aortic nodal dissection for gastric cancer. N Engl J Med. 2008;359:453-62.

6. Japanese Gastric Cancer Association. Japanese classification of gastric carcinoma-2nd English edition-. Gastric Cancer. 1998;1:10-24
7. Tokunaga M, Ohyama S, Hiki N, et al. Therapeutic value of lymph node dissection in advanced gastric cancer with macroscopic duodenum invasion: is the posterior pancreatic head lymph node dissection beneficial? Ann Surg Oncol. 2009;16:1241-6.

8. Sasako M, Sano T, Yamamoto S, et al. Left thoracoabdominal approach versus abdominal-transhiatal approach for gastric cancer of the cardia or subcardia: a randomised controlled trial. Lancet Oncol. 2006;7:644-51.

9. Gotoda T, Yanagisawa A, Sasako M, et al. Incidence of lymph node metastasis from early gastric cancer: estimation with a large number of cases at two large centers. Gastric Cancer. 2000;3: 219-25.

10. Hirasawa T, Gotoda T, Miyata S, et al. Incidence of lymph node metastasis and the feasibility of endoscopic resection for undifferentiated-type early gastric cancer. Gastric Cancer. 2009;12: 148-52.

11. Fukase K, Kato M, Kikuchi S, et al. Effect of eradication of Helicobacter pylori on incidence of metachronous gastric carcinoma after endoscopic resection of early gastric cancer: an openlabel, randomised controlled trial. Lancet. 2008;372:392-7.

12. Murad AM, Santiago FF, Petroianu A, et al. Modified therapy with 5-FU, doxorubicin and methotrexate in advanced gastric cancer. Cancer. 1993;72:37-41.

13. Glimelius B, Hoffman K, Haglund U, et al. Initial or delayed chemotherapy with best supportive care in advanced gastric cancer. Ann Oncol. 1994;5:189-90.

14. Pyrhönen S, Kuitunen T, Nyandoto P, et al. Randomized comparison of fluorouracil, epidoxorubicin and methotrexate (FEMTX) plus supportive care with best supportive care alone in patients with non-resectable gastric cancer. Br J Cancer. 1995;71: 587-91.

15. Koizumi W, Narahara H, Hara T, et al. Randomized phase III study of S-1 alone versus S-1 cisplatin in the treatment for advanced gastric cancer (The SPIRITS trial): a phase III trial. Lancet Oncol. 2008;9:215-21.

16. Boku N, Yamamoto S, Shirao K, et al. Fluorouracil versus combination of irinotecan plus cisplatin versus S-1 in metastatic gastric cancer: a randomized phase3 study. Lancet Oncol. 2009;10:1063-9.

17. Narahara H, Iishi H, Imamura $H$, et al. Randomized phase III study comparing the efficacy and safety of irinotecan plus S-1 with S-1 alone as first-line treatment for advanced gastric cancer (study GC0301/TOP-002). Gastric Cancer. 2011;14:72-80.

18. Shirao K, Boku N, Yamada Y, et al. Randomized phase III study of 5-fluorouracil continuous infusion (5-FUci) versus methotrexate and 5-FU sequential (MF) in gastric cancer with peritoneal metastasis: JCOG 0106. Proc Am Soc Clin Oncol. 2009;27: 4545.

19. Sakuramoto S, Sasako M, Yamaguchi T, et al. Adjuvant chemotherapy for gastric cancer with S-1, an oral fluoropyrimidine. N Engl J Med. 2007;357:1810-20.

20. Huscher CG, Mingoli A, Sgarzini G, et al. Laparoscopic versus open subtotal gastrectomy for distal gastric cancer: five-year results of a randomized prospective trial. Ann Surg. 2005;241: 2132-237.

21. Kitano S, Shiraishi N, Fujii K, et al. A randomized controlled trial comparing open vs laparoscopy-assisted distal gastrectomy for the treatment of early gastric cancer: an interim report. Surgery. 2002;131(1 Suppl):306-11.

22. Macdonald JS, Smalley SR, Benedetti J, et al. Chemoradiotherapy after surgery compared with surgery alone for adenocarcinoma of the stomach or gastroesophageal junction. N Engl J Med. 2001;345:725-30.

23. Cunningham D, Allum WH, Stenning SP, et al. Perioperative chemotherapy versus surgery alone for resectable gastroesophageal cancer. N Engl J Med. 2006;355:11-20. 
24. Nakajima T, Kinoshita T, Nashimoto A, et al. Randomized controlled trial of adjuvant uracil-tegafur versus surgery alone for serosa-negative, locally advanced gastric cancer. $\mathrm{Br}$ J Surg. 2007;94:1468-76.

25. Ajani JA, Winter K, Okawara GS, et al. Phase II trial of preoperative chemoradiation in patients with localized gastric adenocarcinoma (RTOG9904): quality of combined modality therapy and pathologic response. J Clin Oncol. 2006;24:3953-8.

26. Hartgrink HH, Putter H, Kranenbarg EK, et al. Value of palliative resection in gastric cancer. Br J Surg. 2002;89:1438-43. 